\title{
Actividad física y cognición: inseparables en el aula
}

\section{Anya Doherty ${ }^{1 *}$, Anna Forés Miravalles $^{2}$}

\author{
${ }^{1}$ Universitat de Barcelona, España; Pontificia Universidad Católica de Valparaíso, Chile; anyadoher- \\ ty@gmail.com \\ 22Universitat de Barcelona; annafores@ub.edu
}

\section{Resumen}

Tradicionalmente, la educación ha tendido a compartimentar el pensamiento abstracto, la emoción y la actividad física. Sin embargo, la evidencia neurocientífica sugiere que estos tres elementos están estrechamente vinculados con el proceso de aprendizaje. En la "Introducción" de este artículo se repasa el contexto actual: cómo la tradicional clase magistral relega a los estudiantes a un papel pasivo y sedentario que impide el movimiento físico; cómo en los colegios se van reduciendo las horas de recreo y suprimiendo las clases de educación física o aquellas asignaturas que involucran todo el cuerpo (teatro, música, actividades al aire libre), con lo cual se limita aún más la presencia de la actividad física en el entorno de aprendizaje. La evidencia neurocientífica sugiere que el sedentarismo no solo tiene un impacto nocivo en el bienestar físico, sino también en la salud cerebral. El ser humano está diseñado para moverse, para interrelacionarse con su medioambiente, con el movimiento: la actividad física es un factor clave que contribuye al funcionamiento saludable del cerebro. En la sección 2, "Aportes de la investigación neurocientífica", las autoras presentan y analizan diversos estudios y metaanálisis que destacan la asociación positiva entre la actividad física y la cognición en estudiantes de Educación Primaria y Secundaria. En estas investigaciones examinan este vínculo en tres niveles: el incremento de la vascularización (que incrementa el oxígeno y la glucosa en el cerebro); la liberación de neurotransmisores y el factor neurotrófico derivado del cerebro (BDNF en sus siglas en inglés) que favorecen la neurogénesis, la memoria, la atención y la motivación; y el desarrollo de circuitos neurales complejos relacionados con el movimiento y su interconexión con las funciones ejecutivas del cerebro. En la sección 3, "Discusión", se repasan las limitaciones y las aplicaciones de la evidencia examinada. El artículo concluye con unas recomendaciones para que los docentes puedan integrar la actividad física en el aula o en el entorno de aprendizaje. Teniendo en cuenta esta evidencia y la realidad educacional actual, que generalmente considera al aprendizaje como una actividad abstracta, divorciada de nuestra corporalidad, las autoras argumentan la necesidad de incorporar la actividad física al entorno de aprendizaje.

\begin{abstract}
Traditional education has tended to compartmentalize abstract thought, emotion, and physical activity. However, neuroscientific evidence suggests that these are closely interlinked in the learning process. This article, in the Introduction, provides an overview of the current context: the traditional lecture-style lesson relegates students to a passive and sedentary role, precluding physical move-
\end{abstract}


ment; at schools recess hours are being reduced and physical education classes, dropped - as are subjects that involve the whole body (theater, music, outdoor activity) thus further limiting the scope for physical movement within the learning milieu. Neuroscientific evidence suggests that sedentarism impacts negatively on brain health, and not only physical well-being. Humans are designed to be on the move, to interact with their environment through movement: physical activity is a key factor for healthy brain function. In section 2, Contributions from Neuroscience Research, the authors present and analyze evidence from diverse studies and meta-analyses highlighting the positive association between physical activity and cognition in primary and secondary school students. Based on this research, the authors examine the link physical activity-cognition at three levels: increased vascularization increases oxygen and glucose to the brain; the release of neurotransmitters and Brain Derived Neurotrophic Factor (BDNF) which favor neurogenesis, memory, attention and motivation; and the development of complex movement-related neural circuits and their interconnection with the executive brain functions. In section 3, Discussion, the article explores the limitations and applications of the evidence examined. The article concludes with some recommendations for educators integrate physical activity into their classrooms and/ or learning contexts. Based on this evidence and given the current educational reality which generally approaches learning as an abstract activity divorced from our corporality, the authors argue for the need to incorporate physical activity into the learning context.

Palabras clave: actividad física, neuroeducación, BDNF, ejercicio, neurotransmisores, estado físico, neurociencia

\section{Introducción}

Los neurocientíficos Immordino-Yang y Damasio', en su artículo "We feel, therefore we learn" (2007), aluden a una necesidad de cambio de paradigma en la educación, desde Descartes: "Pienso, luego existo" -que supone un enaltecimiento de lo racional y el pensamiento abstracto- al paradigma que reconoce los componentes sociales y emocionales de la cognición. En todas las aulas del ámbito mundial estamos presenciando el creciente reconocimiento de la necesidad de incorporar el aprendizaje socioemocional a la praxis docente; aprendizaje basado en una concepción más amplia del aprendiz como "una persona integral", un ser social con emociones. Si en el quehacer docente, se empieza a reconocer el lugar de las emociones en el aprendizaje, posiblemente el próximo desafío para los educadores será el reconocimiento del cuerpo como elemento clave en el desarrollo y procesamiento cognitivo. La enseñanza y aprendizaje no pueden enfocarse exclusivamente en el cerebro -en la exclusión del cuerpo-. La tradicional clase magistral relega al estudiante a un rol pasivo y sedentario, y descarta el movimiento físico. La tendencia actual en las escuelas es reducir el tiempo asignado para la actividad física, se prioriza un enfoque en las asignaturas académicas y un énfasis en las pruebas estandarizadas, de manera que se exacerba el sedentarismo en niños/as de edad escolar ${ }^{2}$. Un informe de la Organización Mundial de la Salud (OMS) en 2018 estableció que en 2016 un 18,4\% de niños/as y adolescentes (entre 5 y 19 años), o uno de cada cinco, sufrían sobrepeso u obesidad -diez veces más que en las últimas cuatro décadas ${ }^{3}$. Además, la estadística de la OMS de 2016 revela que $81 \%$ de los adolescentes de edad escolar (11-17 años) no realizaban suficiente actividad física ${ }^{4}$. Evidentemente, ello tiene mayores implicaciones para la salud física, así como un impacto en la cognición y el rendimiento académico.

La evidencia neurocientífica sugiere que el sedentarismo no solo entorpece el aprendizaje, sino que va 
en contra del modo en que la anatomía y el cerebro humano han ido evolucionando: desde la perspectiva de la neurobiología evolutiva, autores como Bramble y Lieberman ${ }^{5}$, Lieberman ${ }^{6}$ y Raichlen y Polk ${ }^{7}$ proponen que correr y caminar largas distancias moldearon la anatomía y el tamaño cerebral del ser humano. Vorkapic-Ferreira et al. ${ }^{8}$ plantean que, según esta hipótesis de evolución por carreras en largas distancias, el movimiento desempeñó un rol crucial en el desarrollo de rasgos anatómicos humanos, de manera que esculpió y estructuró el cerebro. Proponen que, el cuerpo humano -incluido el cerebro- ha evolucionado para resistir periodos extendidos de estrés cardiovascular. Explican, además, que diversos estudios han demostrado que el ejercicio aeróbico aumenta la proliferación neuronal -síntesis de factores neurotróficos (como BDNF), gliogénesis, sinaptogénesis- y reduce la inflamación sistemática. Todos estos efectos tienen un impacto significativo en la mejora de la salud mental, reducen el deterioro de la materia gris que se produce con el envejecimiento y mejoran las funciones cognitivas ${ }^{8}$. Estamos diseñados para movernos, nos interrelacionamos con nuestro medio a través del movimiento: la actividad física es el fundamento del funcionamiento cerebral, tal como afirma Llinás ${ }^{9}$, Ratey y Hagerman ${ }^{10}$ y Wolpert ${ }^{11}$. Observan Diamond ${ }^{12}$ y Rosenbaum ${ }^{13}$ que los mismos sistemas o los que se solapan sustancialmente son claves tanto para las funciones cognitivas como motoras. "El cerebro no reconoce la misma aguda división entre función cognitiva y motora (o entre funcionamiento cognitivo y emocional, o funcionamiento social y emocional, etc.) que nosotros imponemos con nuestro pensamiento"14 [traducción de la autora].

Durante la última década, la investigación neurocientífica ha mostrado evidencia significativa de que la actividad física y la cognición están favorablemente entrelazados ${ }^{12,15-21}$. Actualmente, numerosos estudios señalan los beneficios de amplio espectro de la actividad física para la cognición: cambios neurofisiológicos y neuroquímicos que mejoran el funcionamiento cerebral, modifican la estructura cerebral, aportan mayor bienestar y mejoran el aprendizaje. Sin embargo, algunos estudios han obtenido resultados mixtos que señalan una relación entre actividad física y cognición neutra, insignificante o negativa. Así, los investigadores animan al refinamiento de los parámetros de la investigación y a un mayor rigor metodológico para poder identificar variables contextuales implicadas (rango etario de los sujetos, intensidad, duración o tipo de actividad física, etc. $)^{22}$. Globalmente, sin embargo, los educadores tienen por delante el desafío de explorar cómo implementar este conocimiento de la asociación favorable entre actividad física y cognición para mejorar el proceso de enseñanza-aprendizaje.

\section{Aportes de la neurociencia}

¿De qué forma favorece la actividad física a la cognición? Mediante el incremento en el volumen de sangre que riega el cerebro (vascularización), la liberación de neuroquímicos, y los circuitos neuronales. A continuación, y citando una selección de estudios de investigación, se examina cada uno de estos elementos. En primer lugar, el cerebro humano representa solo un $2 \%$ de la masa corporal, pero requiere el $20 \%$ de la energía que consumimos ${ }^{23}$. Con la actividad física, se acrecienta el flujo sanguíneo (vascularización), que a su vez hace aumentar el oxígeno y genera los nutrientes que llegan al cerebro, lo cual fomenta la actividad cerebral ${ }^{24,25}$. Si, en el aula, el docente simplemente pide a sus estudiantes que se pongan de pie y se estiren, el cerebro recibe un $7 \%$ más oxígeno ${ }^{26}$. Entonces, si el docente incorpora actividades de movimiento a su planificación de la clase o intercala breves pausas de movimiento, se está fomentando la actividad cerebral.

Hay numerosos estudios al respecto. Para citar algunos: una investigación con estudiantes de entre 9 y 11 años mostró que con pausas de actividad física con una duración de 4 minutos durante la clase se mejoró la atención selectiva ${ }^{27}$. Otro estudio reveló que una pausa de 10 minutos diarios para realizar movimientos ("energizantes") en clase mejoró la atención y la concentración en el trabajo, sobre todo en estudiantes que habitualmente mostraban dificultades para concentrarse en el trabajo ${ }^{28}$. En un estudio sobre la cognición corporizada, estudiantes universitarios de física que participaron en un experimento sobre las mecánicas de las fuerzas del movimiento de una rueda de bicicleta revelaron mayor activación de regiones sensoriomotoras durante el aprendizaje y la memorización, de modo que lograron resultados notablemente mejores que sus pares sedentarios en una prueba sobre la temática ${ }^{29}$. Estos circuitos sensoriomotores añaden detalle y significado cinético a su proceso de pensamiento ${ }^{30-33}$. Diamond ${ }^{14}$ hace la 
analogía de conducir un automóvil: ¿Cuándo recuerdas mejor la ruta: cuando conduces tú el coche o cuando vas de pasajero? Claramente, cuando los estudiantes tienen un rol activo y multisensorial en su aprendizaje que llega a regiones cerebrales adicionales se fomenta el aprendizaje. La actividad física se puede incorporar en el aula o durante la jornada escolar. Castelli et al. ${ }^{19}$, en un metaanálisis, explican cómo con la incorporación de actividad física en el aula se alcanzan mejores resultados en pruebas y resultados académicos ${ }^{34,35}$. Castelli ${ }^{19}$ afirma también que la incorporación de actividad física vigorosa a la jornada escolar (rutinas de baile, juegos de correr, clases de educación física) mejoró la memoria, la concentración y el rendimiento académico ${ }^{36-39}$.

La actividad física provoca la liberación de neuroquímicos que favorecen el aprendizaje y la memoria. Estos neurotransmisores incluyen dopamina, asociada con la motivación, el enfoque y el aprendizaje; serotonina, que mejora el estado anímico, y norepinefrina, que mejora la atención, la percepción y la motivación ${ }^{40}$. El bienestar emocional es crucial para el aprendizaje. Cuando la amígdala del "sistema emocional" o "sistema límbico" del cerebro detecta estados emocionales como el estrés, el miedo o la rabia, se inunda con niveles excesivos de norepinefrina y dopamina, y se "congela" en el llamado "secuestro de la amígdala"41, por lo que impide el procesamiento de información nueva en la formación de memoria en el hipocampo; en otras palabras, impide el aprendizaje $^{42}$. La corteza prefrontal, donde se concentran las funciones cognitivas superiores (las funciones ejecutivas), es también extremadamente vulnerable a la liberación de norepinefrina y cortisol, inducida por el estrés y que lleva a la disfunción prefrontal ${ }^{43,44}$. Como observan Lavados ${ }^{45}$ y Mora ${ }^{46}$, aprendemos lo que amamos: las emociones positivas abren camino para el aprendizaje. 0, parafraseando a Amanda Céspedes $^{47}$, para el aprendizaje efectivo necesitamos aprendizaje afectivo. Efectivamente, los estados emocionales negativos que experimentan los estudiantes interfieren en el aprendizaje. $Y$, al contrario, si el profesor es consciente del estado anímico de sus estudiantes y trabaja para cultivar en el aula un entorno positivo y seguro en términos socioemocionales, se realzan el aprendizaje y la memoria ${ }^{14}$. Por lo tanto, cuando los docentes incorporan actividad física al proceso de aprendizaje, con la consecuente liberación de los neurotransmisores explicados arriba, están fomentando potencialmente estados anímicos positivos, reducen el estrés y favorecen el aprendizaje y la memoria ${ }^{42,45}$.

Además, dado que las actividades de movimiento normalmente incluyen interacción y contacto visual, se estimula el "cerebro social", de modo que provoca la activación de las neuronas espejo, que favorecen la empatía; la liberación de endorfinas que proporcionan una sensación de bienestar; el neurotransmisor de la vinculación social, la oxitocina, y neurotransmisores motivacionales como la dopamina ${ }^{48,49}$. Diamond y Ling ${ }^{50}$ enfatizan que nuestro bienestar emocional, social y físico impactan fuertemente sobre la cognición. Explican cómo las funciones ejecutivas son las primeras en sufrir si el individuo se siente estresado, triste, solo, en mal estado físico, o con falta de sueño ${ }^{50}$. El ejercicio físico combate estos estados dañinos, pues facilita las funciones cognitivas superiores $^{51-53}$ y mejora el sueño $0^{54-56}$. Gould ${ }^{57}$ señala el doble impacto de la actividad física: reduce el estrés y las consecuencias negativas que tiene para el funcionamiento cerebral, mientras que favorece la neurogénesis y el rendimiento cerebral.

Dado que los estudiantes están habitualmente expuestos al estrés (las exigencias de pruebas, plazos límite, etc.), la actividad física puede tener un rol de reductor del mismo, que consecuentemente mejora la capacidad del cerebro para el procesamiento cognitivo $^{46}$. Las investigaciones también resaltan la importancia de que las actividades de movimiento sean voluntarias y se realicen con alegría (la actitud del estudiante hacia la actividad importa): Cuando la actividad física es colaborativa, no amenazante, y divertida (versus obligación aburrida), se aumenta la función cerebral ${ }^{50}$. Ratey y Hagerman ${ }^{10}$ presentan el estudio de caso de una escuela secundaria estadounidense que integraba en la jornada escolar sesiones de educación física que eran motivacionales, divertidas y no competitivas. Cuando la escuela tomó la prueba internacional de estándares, Trends in International Mathematics and Science Study (TIMSS), alcanzó el primer lugar en el mundo en ciencia y el sexto lugar mundial en matemática. El ranking promedio de Estados Unidos es el lugar 18 o 19. Aunque estos datos parecen alentadores, cabe mencionar que este caso fue un cambio en la escuela, un cambio en la política sobre actividad física, no un experimento dirigido por un equipo de investigación. Así, esta evidencia, aunque potencialmente positiva, se debe tomar 
con cautela; habría que realizar estudios rigurosos de seguimiento para poder reducir las variables en juego y aclarar la interpretación de estos resultados.

El impacto favorable del ejercicio físico voluntario sobre el tamaño y función del hipocampo (región asociada con el aprendizaje) en roedores ha sido ampliamente estudiado ${ }^{58,59}$. El ejercicio aeróbico voluntario es un gatillo excelente del factor neurotrófico derivado del cerebro (BDNF, sus siglas en inglés), que estimula el crecimiento de nuevas neuronas (neurogénesis), las nuevas conexiones entre neuronas (sinaptogénesis) y la protección de circuitos neuronales existentes en el hipocampo ${ }^{40,60,61}$. En adultos mayores, la liberación de BDNF contrarresta el encogimiento hipocampal ${ }^{60}$-un campo de investigación pujante, pero que queda fuera del alcance de este artículo-. Es notable que la actividad física causa estos cambios estructurales, particularmente en la parte del cerebro donde ocurre el aprendizaje, y no en las regiones sensoriales ni motoras. Ratey ${ }^{10}$ explica que tiene sentido evolutivo: "La razón por la que necesitamos una capacidad de aprender es para ayudarnos a encontrar, obtener y almacenar comida. Necesitamos comida para aprender y necesitamos el aprendizaje para encontrar la fuente del combustible" [traducción de la autora]. Como plantean Gómez-Pinilla et al. ${ }^{62}$, estos hallazgos sugieren que el BDNF es parte de un mecanismo central a través del cual la actividad física se integra con elementos de metabolismo de energía para impactar en elementos de la función hipocampal. Efectivamente, la actividad física hace crecer el cerebro con la liberación de BDNF y el aumento de tamaño del hipocampo.

Consideremos algunos de estos cambios estructurales: la investigación revela que los individuos que practican ejercicio físico tienen mayor masa cortical $^{63}$. Los niños que son más activos tienen mayor volumen de materia gris y blanca. El hipocampo, región cerebral donde los aprendizajes se convierten en memoria, es mayor en niños/as que tienen mejor estado físico; también son mayores los ganglios basales, estructuras cerebrales asociadas al aprendizaje $^{10,64}$. Los niños de ese estudio mostraron mejor rendimiento académico en tareas relacionadas con las funciones ejecutivas y la memoria asociativa ${ }^{19}$. Chaddock-Heyman et al. ${ }^{2}$ muestran evidencias de que los niños más activos experimentan cambios en su materia blanca (cuerpo calloso), que integra información cognitiva, motora y sensorial entre el he- misferio derecho e izquierdo. Los individuos en mejor estado físico muestran diferencias en la estructura y función cerebrales ${ }^{17,19}$. Respecto a la función cerebral, los estudios señalan mayor conectividad entre hipocampo, región prefrontal y cingulado ${ }^{2}$. Los resultados académicos o el desempeño cognitivo muestran mejoras relacionadas con la actividad física $^{18-20,35,37,65}$.

Un estudio longitudinal de López-Vicente ${ }^{66}$ (el primero de este tipo) siguió a 1.400 niños y niñas desde la edad de 6 años hasta la adolescencia. Las conclusiones indicaron que los sujetos con menores niveles de actividad física a la edad de 6 años mostraron resultados significativamente peores en pruebas de memoria cuando eran adolescentes, en comparación a sus pares que tenían mejor estado físico. Este dato parece sugerir que niveles más altos de actividad física durante los años de desarrollo del niño tienen beneficios cognitivos a largo plazo. Los estudios de investigación de Hillman et al. ${ }^{17}$ muestran que niños/ as con mayor nivel de fitness aeróbico mostraron mejor función neurocognitiva, evidenciada en niveles mayores de atención y memoria de trabajo, así como la rapidez de respuesta en pruebas cognitivas. El metaanálisis de Sibley y Etnier ${ }^{67}$ evidencia que la actividad física mejora las capacidades perceptuales, $\mathrm{Cl}$, desempeño, pruebas verbales, pruebas de matemática, entre otros. En los estudios citados anteriormente, es importante destacar que no se debe suponer la causalidad entre las variables, como el nivel de fitness y las distintas funciones cerebrales medidas. Estos resultados señalan una relación favorable, pero los matices de esa relación y de otras variables merecen mayor investigación.

La investigación neurocientífica está desvelando una mayor interconectividad entre las regiones cerebrales que antes se imaginaba: por ejemplo, las funciones ejecutivas (la inhibición y control de la interferencia, flexibilidad cognitiva, memoria de trabajo ${ }^{68}$ asociadas con la corteza prefrontal están estrechamente vinculadas con otras múltiples regiones; por ejemplo, las áreas asociadas con la emoción y el movimiento físico ${ }^{12,14}$. La investigación revela que el cerebelo, que hasta hace muy poco se relacionaba solo con las funciones motoras, está también vinculado con el pensamiento, la atención, las emociones $y$ las destrezas sociales ${ }^{12}$. El cerebelo desempeña un rol activo en las funciones cognitivas, no solo en las motoras. La percepción de que el desarrollo motor y 
el cognitivo estén separados ya no convence, a la luz de esta evidencia. De hecho, tanto con la actividad física como con el procesamiento del pensamiento hay una coactivación del cerebelo y la corteza prefrontal. El daño al cerebelo impacta sobre el procesamiento cognitivo y el daño a la corteza prefrontal impacta sobre las funciones motoras ${ }^{12}$. Los circuitos cerebrales involucrados en el movimiento físico no son completamente distintos a los que se emplean para el pensamiento. Como observa Llinás ${ }^{9}$, el proceso que llamamos "pensar" es la internalización evolutiva del movimiento. 0 , parafraseando a Ratey ${ }^{10,69}$, la naturaleza es frugal: cuando hacemos ejercicio, particularmente con movimientos complejos y secuenciados, empleamos circuitos neuronales para predecir, secuenciar, estimar, planificar, ensayar, autoobservar, juzgar, corregir errores, ajustar tácticas y recordar. Estos circuitos son los mismos que empleamos para el pensamiento abstracto ${ }^{68}$.

Pero ¿hasta qué punto son transferibles las funciones ejecutivas? Diamond y Ling ${ }^{50} \mathrm{lo}$ analizan en un metaanálisis rigoroso, y llegan a la conclusión de que la transferencia de funciones ejecutivas es estrecha. En otras palabras, si con la actividad física se ejercita la memoria del trabajo, esta función ejecutiva se transfiere a la actividad cognitiva, y no a otras como la flexibilidad cognitiva o inhibición del impulso, dado que no se ejercitaron. Diamond y Ling notaron también que los individuos con las funciones ejecutivas más débiles se benefician más de esta transferencia. Concluyen que la actividad física que implica desafíos cognitivos, como planificación, concentración, inhibición del impulso, flexibilidad cognitiva, memoria de trabajo (artes marciales, deportes de equipo o yoga), contribuyen a mejorar estas funciones ejecutivas en desafíos cognitivos ${ }^{50,68,70-72}$. La evidencia indica que la actividad física consciente, o mindful, fomenta las funciones ejecutivas, mientras que la actividad física sin sentido o inconsciente no lo hace ${ }^{68,73,74}$. Por lo tanto, actividad física y cognición están estrechamente entrelazadas, y se impactan y coactivan recíprocamente.

\section{Discusión}

Es de suma importancia resaltar las limitaciones en este campo de investigación. Aunque la literatura existente confirma la asociación favorable entre actividad física (también ejercicio físico, que implica que la actividad se repita sistemáticamente) y cognición, diversos metaanálisis ${ }^{40,67,75-77}$ |laman a una cautelosa y crítica interpretación de la investigación en esta área relativamente nueva. A pesar de la asociación positiva entre actividad física y cognición, algunos estudios señalan que este impacto favorable es mínimo y que los resultados están influidos por una multiplicidad de factores y varían según la duración de la intervención de actividad física, la frecuencia, si es aeróbica o no, crónica o puntual, el grado de esfuerzo implicado y si es mindful o mindless ${ }^{35,40,76}$. Cabe mencionar algunas advertencias sobre el impacto de la actividad física puntual (de una sola sesión), así como el impacto de la actividad física crónica sobre el cerebro, y la variabilidad de estos resultados ${ }^{50,76}$. Basso y Suzuki ${ }^{40}$ revisaron 273 estudios para concluir que el impacto de la actividad física puntual es de corta duración. Generalmente se observa en la mejora del estado de ánimo, la reducción del estrés, la presencia extendida de dopamina y serotonina, $y$ un aumento de la actividad hipocampal. A largo plazo, el ejercicio habitual de intensidad moderada a alta con un componente cualitativo o cognitivo tiene un impacto más profundo, de más larga duración en la estructura y función del cerebro y en los circuitos neuronales ${ }^{50,76}$. Otros estudios muestran que la actividad física no perjudica el desempeño académico, dicho de otra forma, su impacto no es positivo ni negativo ${ }^{22,34,78}$. Otro factor de complejidad es el tipo de herramienta empleada para medir el impacto de la actividad física sobre la cognición (EEG, IRMf, rendimiento académico, pruebas cognitivas como Stroop, Go/No Go), y los aspectos que se miden: cambios neurofisiológicos, neuroquímicos o de comportamiento ${ }^{76,77}$

Es necesario estandarizar las variables medidas en los estudios sobre actividad física: su duración, intensidad, percepción de esfuerzo -algo que Basso y Suzuki ${ }^{40}$ proponen con su "índice de ejercicio"para comprender mejor y relativizar los resultados de investigaciones. Finalmente, Diamond y Ling ${ }^{50}$ advierten que debemos ser cautelosos con suponer relaciones causales: aunque numerosos estudios muestran que los individuos con mejor estado físico tienen mayor función cognitiva (por ejemplo, las funciones ejecutivas), esto no implica necesariamente una relación de causa-efecto. Los autores citados anteriormente observan que podría ser que los individuos que optan por una vida más activa lo hacen 
debido a una predisposición cerebral. Hay una llamada de los investigadores en este campo para el desarrollo de herramientas de medición estandarizadas, la aplicación del rigor extremo en investigaciones futuras y el uso de la prudencia en la interpretación de resultados existentes y las generalizaciones que se llevan a cabo $40,50,67,75,77$. Es necesario resaltar lo dañino que puede ser establecer generalizaciones amplias sobre la actividad física y la cognición, pues puede contribuir a la promulgación de los neuromitos. Un neuromito es una creencia sobre el cerebro que se basa en información imprecisa y se populariza (a menudo con intereses comerciales subyacentes). Puede incluso interferir en el proceso de aprendizaje y llegar a impedirlo ${ }^{79}$.

Dicho esto, las conclusiones globales que emergen de la investigación sobre actividad física y cognición señalan una asociación favorable. En el contexto actual, donde las escuelas promueven hábitos sedentarios $^{35}$, la evidencia emergente sobre la interconexión positiva entre actividad física y cognición debería animar a los docentes a integrar la actividad física en sus clases, y a las instituciones educativas a fomentar la actividad física mediante cambios curriculares, infraestructurales y culturales. Alrededor del mundo, distintos establecimientos están implementando cambios, desde las escuelas primarias a Harvard University. Hoy en día, internet está repleto de sugerencias prácticas y programas como los usados en los estudios de investigación mencionados más arriba. Hay que animar a los estudiantes a que lleguen al colegio a pie o en bicicleta, salvaguardar el tiempo del recreo, fomentar recreos y clases de educación física activas y entretenidas, y concienciar a madres, padres y docentes de que se puede avanzar hacia una experiencia de aprendizaje más integrada y holística, que trate al niño en su conjunto (socioemocional, cognitivo y físico). El profesorado puede integrar actividades de movimiento en distintos momentos del día: cuando los niveles de energía caen después de mediodía, cuando la capacidad atencional se agota, para romper el hielo, provocar la curiosidad, construir la confianza en el grupo y reducir el estrés. Los recursos recomendados aquíro pueden servir como un punto de partida para docentes que quieren iniciar un cambio para llegar a clases más activas. Además, cabe observar que cuando los docentes creen en la importancia de no excluir la corporalidad de los procesos cognitivos se acostumbran a aprovechar las oportunidades que se presentan en aula para incluir la actividad física en beneficio del aprendizaje. Las clases se pueden empezar con una actividad de estiramientos, los trabajos en grupo se pueden organizar de forma que los estudiantes necesiten moverse por diferentes estancias del aula, se pueden realizar trabajos en afiches en las distintas paredes, organizar actividades en equipo que implican correr a la pizarra a escribir o pegar algo, tirar la pelota $u$ otro objeto entre pares mientras hacen actividades de memoria, el juego Simon Says (el monito mayor), mímica, teatro, coreografías cortas, etc. Los niños y las niñas (también los adultos) necesitan moverse. Cuando los docentes reconocen y honran esta necesidad en el aula y en la escuela, los aprendices están mejor posicionados para disfrutar y tener éxito en el aprendizaje.

\section{Conclusiones}

Para concluir, este trabajo ha intentado ofrecer un esbozo inicial del volumen creciente de estudios que afirman la interconectividad entre actividad física y cognición. Aunque muchas investigaciones señalan la asociación positiva entre actividad física, estado físico y cognición, se requiere una aproximación cautelosa, como hemos explicitado más arriba. Tal como advirtió el neurocientífico John Bruer $^{81}$ en su artículo "Brain and education: a bridge too far", los educadores deben ser precavidos a la hora de emitir generalizaciones amplias basadas en hallazgos iniciales o información superficial. Hoy en día, la educación presencia un importante cambio de paradigma. De un paradigma antiguo que elevaba al pensamiento abstracto, a un paradigma que concibe la indivisibilidad de lo social, emocional y físico en el aprendizaje. Esta concepción holística del ser humano no es novedosa: los griegos tenían una idea clara de la interconectividad entre cerebro, emoción y cuerpo. En la actualidad, la investigación neurocientífica parece confirmar las ideas de los grandes pensadores en educación: Vygotsky, Piaget, Freire y Montessori. Su intuición de que el aprendizaje es un proceso social, emocional, interactivo, significativo, físico y constructivo se está validando hoy mediante la investigación neurocientífica. Este trabajo ha examinado algunas de las contribuciones claves de la neurociencia sobre la conexión entre actividad física y cognición, con la esperanza de aportar cambios en la praxis docente. 


\section{Referencias}

1. Immordino-Yang M, Damasio A. We feel, therefore we learn: the relevance of affective and social neuroscience to education. Mind Brain Educ. 2007; 1, 3-10. doi: 10.1111/j.1751228X.2007.00004.x.

2. Chaddock-Heyman L, Erickson KI, Kienzler C, Drollette ES, Raine LB, Kao SC et al. Physical activity increases white matter microstructure in children. Front. Neurosci. 2018; 12, 950. Doi: 10.3389/fnins.2018.00950.

3. Organización Mundial de la Salud. World Health Statistics 2018: Monitoring health for the SDGs. Disponible en: https://www. who.int/gho/publications/world_health_statistics/2018/en/ (consultado el 01/06/2020).

4. Organización Mundial de la Salud. Prevalence of insufficient physical activity among school going adolescents. Disponible en: https://apps.who.int/gho/data/view.main.2463AD0?lan$\mathrm{g}=$ en\# (consultado el 01/06/2020).

5. Bramble DM, Lieberman DE. Endurance running and the evolution of homo. Nature. 2004; 432, 345-352. doi: 10.1038/ nature03052.

6. Lieberman DE. Four legs good, two legs fortuitous: brains, brawn and the evolution of human bipedalism. En: Losos JC editor. Light of evolution. Greenwood Village, CO: Roberts and Company; 2010. p. 55-71.

7. Raichlen DA, Polk JD. Linking brains and brawn: exercise and the evolution of human neurobiology. Proc. R. Soc. B. 2013; 280, 20122250. doi: 10.1098/rspb.2012.2250.

8. Vorkapic-Ferreira C, Souza Góis R, Gomes LP, Britto A, Afrânio $B$, Dantas $M$ et al. Nascidos para correr: a importância do exercício para a saúde do cerebro. Born to run: the importance of exercise for the brain health. Rev. Bras. Med. Esporte. 2017; 23, 495-503. doi: 10.1590/1517-869220172306175209.

9. Llinás R. I of the vortex: from neurons to self. Cambridge, MA: MIT Press; 2001.

10. Ratey J, Hagerman E. Spark: the revolutionary new science of exercise and the brain. Nueva York, NY: Little, Brown \& Company; 2008

11. Wolpert $D$. The real reason for brains - Is movement. Cambridge University Research News. 2011. Disponible en: https:// www.cam.ac.uk/research/news/the-man-with-the-goldenbrain. Consultado el 13/06/2020.

12. Diamond A. Close interrelation of motor development and cognitive development and of the cerebellum and prefrontal cortex. Child Dev. 2000; 71, 44-56. doi: 10.1111/14678624.00117.

13. Rosenbaum DA, Carlson RA, Gilmore RO. Acquisition of intellectual and perceptual-motor skills. Annu. Rev. Psychol. 2001; 52, 453-470.

14. Diamond, A. The evidence base for improving school outcomes by addressing the whole child and by addressing skills and attitudes, not just content. Early Educ. Dev. 2010; 21, 780793. doi: $10.1080 / 10409289.2010 .514522$.

15. Cotman CW, Berchtold NC. Exercise: a behavioral intervention to enhance brain health and plasticity. Trends Neurosci. 2002; 25, 295-301. doi: 10.1016/S0166-2236(02)02143-4.

16. Hillman $\mathrm{CH}$, Castelli DM, Buck SM. Aerobic fitness and neu- rocognitive function in healthy preadolescent children. Med. Sci. Sports Exerc. 2005; 37, 1967-1974. doi: 10.1249/01. mss.0000176680.79702.ce.

17. Hillman $\mathrm{CH}$, Buck S, Themanson J, Pontifex M, Castelli, D. Aerobic fitness and cognitive development: event-related brain potential and task performance indices of executive control in preadolescent children. Dev.Psychol. 2009a; 45, 114-129. doi: $10.1037 / a 0014437$.

18. Chaddock L, Erickson K, Prakasch RS, Kima J., Voss M, Pontifex $M$. et al. A neuroimaging investigation of the association between aerobic fitness, hippocampal volume, and memory performance in preadolescent children. Brain Res. 2010; 1358, 172-183. doi: 10.1016/j.brainres.2010.08.049.

19. Castelli D, Barcelona J, Glowacki E, Calvert H. Active education: Growing evidence on physical activity and academic performance. San Diego, CA: Active Living Research; 2014. Disponible en: https://www.researchgate.net/publication/269708986 (consultado el 13/06/2020).

20. Erickson K, Hillman C, Kramer A. Physical activity, brain, and cognition. Curr. Opin. Behav. Sci. 2015; 4, 27-32. doi: 10.1016/j.cobeha.2015.01.005.

21. Mandolesi L, Polverino A, Montuori S, Foti F, Ferraioli G, Sorrentino $P$, et al. Effects of physical exercise on cognitive functioning and wellbeing: biological and psychological benefits. Front. Psychol. 2018; 9, 509. doi: 10.3389/fpsyg.2018.00509.

22. Marques A, Gómez F, Martins J, Catunda R, Sarmento H. Association between physical education, school-based physical activity, and academic performance: a systematic review. Retos. 2017; 31, 316-320. Disponible en: https://recyt.fecyt.es/ index.php/retos/article/view/53509.

23. Hart L. How the brain works. Nueva York, NY: Basic Books; 1975.

24. Delp MD, Armstrong RB, Godfrey DA, Laughlin MH, Ross CD, Wilkerson MK. Exercise increases blood flow to locomotor, vestibular, cardiorespiratory and visual regions of the brain in miniature swine. J. Physiol. 2001; 533, 849-859. doi: 10.1111/ j.1469-7793.2001.t01-1-00849.x.

25. Hillman $\mathrm{CH}$, Pontifex MB, Raine LB, Castelli DM, Hall EE, Kramer AF. The effect of acute treadmill walking on cognitive control and academic achievement in preadolescent children. Neuroscience. 2009b; 159, 1044-1054. doi: 10.1016/j. neuroscience.2009.01.057.

26. Krock LP, Hartung GH. Influence of post-exercise activity on plasma catecholamines, blood pressure and heart rate in normal subjects. Clin. Autonom. Res. 1992; 2, 89. doi: 10.1007/ BF01819663.

27. Ma J, Le Mare L, Gurd B. Four minutes of in-class high-intensity interval activity improves selective attention in 9- to 11-year olds. Appl. Physiol. Nutr. Metab. 2015; 40, 238-244. doi: 10.1139/apnm-2014-0309.

28. Mahar M, Murphy S, Rowe D, Golden J, Shields T, Raedke T. Effects of a classroom-based program on physical activity and on-task behavior. Med. Sci. Sports Exerc. 2006; 38, 20862094. doi: 10.1249/00005768-200605001-01239.

29. Kontra C, Lyons DJ, Fischer SM, Beilock SL. Physical expe- 
rience enhances science learning. Psychol. Sci. 2015; 26, 737749. doi: $10.1177 / 0956797615569355$.

30. Glenberg AM. What memory is for target article and commentaries]. Behav. Brain Sci. 1997; 20, 1-55. doi: 10.1017/ S0140525X97000010.

31. Barsalou LW, Simmons WK, Barbey AK, Wilson CD. Grounding conceptual knowledge in modality-specific systems. Trends Cognit. Sci. 2003; 7, 84-91. doi: 10.1016/S13646613(02)00029-3.

32. Zwann RA, Taylor LJ. Seeing, acting, understanding: motor resonance in language comprehension. J. Exp. Psychol. Gen. 2006; 135, 1-11. doi: 10.1037/0096-3445.135.1.1.

33. Beilock SL, Lyons IM, Mattarella-Micke A, Nusbaum HC y Small SL. Sports experience changes the neural processing of action language. Proc. Natl. Acad. Sci. U.S.A. 2008; 105, 1326913273. doi: 10.1073/pnas.0803424105.

34. Ahamed Y, Macdonald H, Reed K, Naylor P, Liu-Ambrose T, McKay H. School-based physical activity does not compromise children's academic performance. Med. Sci. Sport. Exerc. 2007; 39, 371-376. doi: 10.1249/01.mss.0000241654.45500.8e.

35. Donnelly JE, Lambourne K. Classroom-based physical activity, cognition, and academic achievement. Prev. Med. 2011; 52, S36-S42. doi: 10.1016/j.ypmed.2011.01.021.

36. Carlson SA, Fulton JE, Lee SM, Maynard LM, Brown DR, Kohl, $\mathrm{HW}$, et al. Physical education and academic achievement in elementary school: data from early childhood longitudinal study. Am. J. Public Health. 2008; 98, 721-727. doi: 10.2105/ AJPH.2007.117176.

37. Pesce C, Crova C, Cereatti L, Casella R, Bellucci M. Physical activity and mental performance in preadolescents: effects of acute exercise on free-recall memory. Ment. Health Phys. Act. 2009; 2, 16-22. doi: 10.1016/j.mhpa.2009.02.001.

38. Castelli DM, Hillman $\mathrm{CH}$, Hirsch J, Hirsch A, Drollette E. FIT Kids: time in target heart zone and cognitive performance. Prev Med. 2011; 52, S55-S59. doi: 10.1016/j.ypmed.2011.01.019.

39. Gao Z, Hannan P, Xiang P, Stodden DF, Valdez VE. Video game-based exercise, Latino children's physical health, and academic achievement. Am. J. Prev. Med. 2013; 44, S240-S246. doi: 10.1016/j.amepre.2012.11.023.

40. Basso JC, Suzuki WA. The effects of acute exercise on mood, cognition, neurophysiology, and neurochemical pathways: a review. Brain Plasticity. 2017; 2, 127-152. doi: 10.3233/BPL160040.

41. Goleman D. Emotional intelligence: Why it can matter more than IQ. Nueva York, NY: Bantam Books; 2005

42. Willis, J. What You Should Know About Your Brain. Educational Leadership ASCD. 2009. Disponible en: http://www.ascd. org/ASCD/pdf/journals/ed_lead/el200912_willis.pdf.

43. Birnbaum S, Gobeske KT, Auerbach J, Taylor JR, Arnsten AFT. A role for norepinephrine in stress-induced cognitive deficits: alpha-1-adrenoceptormediation in the prefrontal cortex. Biol. Psychiatry. 1999; 46, 1266-1274. doi: 10.1016/ S0006-3223(99)00138-9.

44. Liston C, McEwen BS, Casey BJ. Psychosocial stress reversibly disrupts prefrontal processing and attentional control.
Proc. Nat. Acad. Sci. U.S.A. 2009; 106, 912-917. doi: 10.1073/ pnas.0807041106.

45. Lavados J. El cerebro y la educación. Neurobiología del aprendizaje. Santiago de Chile: Taurus; 2012

46. Mora F. Neuroeducación. Madrid: Alianza; 2017.

47. Céspedes A. Educar las emociones. Educar para la vida. Santiago de Chile: Vergara; 2008.

48. Rilling JK, Gutman DA, Zeh TR, Giuseppe P, Bern GS, Kilts CD. A neural basis for social cooperation. Neuron. 2002; 35, 395405. doi: 10.1016/S0896-6273(02)00755-9.

49. Willis J. Cooperative learning is a brain turn-on. Middle School J. 2008; 38, 4-13. doi: 10.1080/00940771.2007.11461587.

50. Diamond A, Ling D. Conclusions about interventions, programs, and approaches for improving executive functions that appear justified and those that, despite much hype, do not. Dev. Cognit. Neurosci. 2015; 18, 34-48. doi: 10.1016/j. den.2015.11.005.

51. Carmack C, de Moor C, Boudreaux E, Amaral-Melendez M, Brantley P. Aerobic fitness and leisure physical activity as moderators of the stress-illness relation. Ann. Behav. Med. 1999; 21, 251-257. doi: 10.1007/BF02884842.

52. Williamson D, Dewey A, Steinberg $\mathrm{H}$. Mood change through physical exercise in nine-to ten-year-old children. Percept. Mot. Skills. 2001; 93, 311-316. doi: 10.2466/pms.2001.93.1.311.

53. Haslacher H, Michlmayr M, Batmyagmar D, Perkmann T, Ponocny-Seliger E, Scheichenberger V, et al. Physical exercise counteracts genetic susceptibility to depression. Neuropsychobiology. 2015; 71, 168-175. doi: 10.1159/000381350.

54. Yang PY, Ho KH, Chen HC, Chien MY. Exercise training improves sleep quality in middle-aged and older adults with sleep problems: a systematic review. J. Physiother. 2012; 58, 157 163. doi: 10.1016/S1836-9553(12)70106-6.

55. Chen LJ, Fox KR, Ku PW, Chang YW. Effects of aquatic exercise on sleep in older adults with mild sleep impairment: a randomized controlled trial. Int. J. Behav. Med. 2015; 23, 501 506. doi: 10.1007/s12529-015-9492-0.

56. Wachob D, Lorenzi DG. Brief report: influence of physical activity on sleep quality in children with autism. J. Autism Dev. Disord. 2015; 45, 2641-2646. doi: 10.1007/s10803-015-2424-7.

57. Gould E. Adult neurogenesis a substrate for experience dependent change. Cell Press. 2015; 19, 151-161. doi: 10.1016/j. tics.2015.01.001.

58. Van Praag H, Shubert T, Zhao C, Gage FH. Exercise enhances learning and hippocampal neurogenesis in aged mice. J. Neurosci. 2005; 25, 8680-8685. doi: 10.1523/JNEUROSCl.1731-05.2005

59. Vivar C, Potter MC, van Praag H. All about running: synaptic plasticity, growth factors and adult hippocampal neurogenesis. Curr. Top. Behav. Neurosci. 2013; 15, 189-210. doi: 10.1007/7854_2012_220.

60. Voss M., Erickson KI, Prakash RS, Chaddock L, Kim JS, Alves $\mathrm{H}$, et al. Neurobiological markers of exercise-related brain plasticity in older adults. Brain Behav. Immun. 2013; 28, 90 99. doi: 10.1016/j.bbi.2012. 10.021.

61. Jeon $\mathrm{YK}, \mathrm{Ha} \mathrm{CH}$. Expression of brain-derived neurotrophic factor, IGF-1and cortisol elicited by regular aerobic exerci- 
se in adolescents. J. Phys. Ther. Sci. 2015; 27, 737-741. doi: 10.1589/jpts.27.737.

62. Gómez-Pinilla F, Vaynman S, Ying Z. Brain-derived neurotrophic factor functions as a metabotrophin to mediate the effects of exercise on cognition. Eur. J. Neurosci. 2008; 28, 2278-2287. doi: 10.1111/j.1460-9568.2008.06524.x.

63. Anderson BJ, Eckburg PB, Relucio KI. Alterations in the thickness of motor Cortical sub-regions after motor-skill learning and exercise. Learn. Mem. 2002; 9, 1-9. doi: 10.1101/ Im.43402.

64. Chaddock L, Erickson K, Prakasch RS, VanPatter M, Voss M, Pontifex $\mathrm{M}$, et al. Basal ganglia volume is associated with aerobic fitness in preadolescent children. Dev. Neurosci. 2010; 32(3), 249-56. doi: 10.1159/000316648.

65. Rama Kranthi T, Syamala E, Amrutha Kumari K, Soni S, Nazeer M. Effect of physical training on short term memory in school going rural children. J. Med. Sci. Res. 2014; 2, 228230. Disponibe en: https://www.researchgate.net/publication/329917067_Effect_of_physical_training_on_short_ term_memory_in_school_going_rural_children.

66. López-Vicente M, Garcia-Aymerich J, Torrent-Pallicer J, Forns $\mathrm{J}$, Ibarluzea J, Lertxundi $\mathrm{N}$, et al. Are early physical activity and sedentary behaviors related to working memory at 7 and 14 years of age? J. Pediatr. 2017; 188, 35-41, e1. doi: 10.1016/j. jpeds.2017.05.079.

67. Sibley BA, Etnier JL. The relationship between physical activity and cognition in children: a meta-analysis. Ped. Exerc. Sci. 2003; 15, 243-256. doi: 10.1123/pes.15.3.243.

68. Diamond A. Activities and programs that improve children's executive functions. Curr. Direct. Psychol. Sci. 2012; 21, 335341. doi: $10.1177 / 0963721412453722$.

69. Ratey J. A user's guide to the brain. Londres: Abacus; 2001.

70. Manjunath NK, Telles S. Improved performance in the Tower of London test following yoga. Indian J. Physiol. Pharmacol. 2001; 45, 351-354.

71. Lakes KD, Hoyt WT. Promoting self-regulation through school-based martial arts training. J. Appl. Dev. Psychol. 2004; 25, 283-302. doi: 10.1016/j.appdev.2004.04.002.

72. Chang YK, Tsai YJ, Chen TT, Hung TM. The impacts of coordinative exercise in executive function in kindergarten children: an ERP study. Exp. Brain Res. 2013; 225, 187-196. doi: 10.1007/s00221-012-3360-9.

73. Oswald WD, Gunzelmann T, Rupprecht R, Hagen B. Differential effects of single versus combined cognitive and physical tra- ining with older adults: the SimA study in a 5-year perspective. Eur. J. Aging. 2006; 3, 179-192. doi: 10.1007/s10433-0060035-z.

74. Moreau D, Morrison AB, Conway ARA. An ecological approach to cognitive enhancement: complex motor training. Acta Psychol. 2015; 157, 44-55. doi: 10.1016/j.actpsy.2015.02.007.

75. Lees C, Hopkins J. Effect of aerobic exercise on cognition, academic achievement and psychosocial function in children. A systematic review of randomized control trials. Prev. Chronic Dis. 2013; 10, E174. doi: 10.5888/pcd10.130010.

76. Tomporowski PD, McCullick B, Pendleton BN, Pesce C. Exercise and children's cognition: the role of exercise characteristics and a place for metacognition. J. Sport Health Sci. 2015; 4, 47-55. Doi: 10.1016/j.jshs.2014.09.003.

77. Donnelly J E, Hillman CH, Castelli D, Etnier J, Lee S, Tomporowski $\mathrm{P}$, et al. Physical activity, fitness, cognitive function, and academic achievement in children: a systematic review. Med. Sci. Sports Exerc. 2016; 48, 1197-1222. doi: 10.1249/ MSS.0000000000000901.

78. Resaland GK, Aadland E, Moe VF, Aadland KN, Skrede T, Stavnsbo M, et al. Effects of physical activity on schoolchildren's academic performance: the Active Smarter Kids (ASK) cluster-randomized controlled trial. Prev. Med. 2016; 91, 322328. doi: 10.1016/j.ypmed.2016.09.005.

79. Dekker S, Lee N, Paul HJ, Jelle J. Neuromyths in education: prevalence and predictors of misconceptions among teachers. Front. Psychol. 2012; 3, 429. doi: 10.3389/ fpsyg.2012.00429.

80. NC Healthy Schools: <www.nchealthyschools.org>; Be Active North Carolina, Inc: <www.beactivenc.org $>$; NC Physical Education for Me: <www.ncpe4me.co>; NC Health and WeIlness Trust Fund: <www.fitkidsnc.com>; ECU Activity Promotion Lab: <www.ecu.edu/cs-hhp/exss/apl.cfm>; Action for Healthy Kids: Tools for Schools: <http://www.actionforhealthykids.org/tools-for-schools/1252-brainbreaks-instant-recess-and-energizers >; NC Public Schools: <www. ncpublicschools. org/curriculum/health $>$; $\langle$ http://www. theteachersguide.com/ClassManagement.htm>; <http:// www.teachervision.fen.com/>; <http://drwilliampmartin. tripod.com/classm.html>. Elige Educar: <https://eligeeducar.cl/15-pausas-cerebrales-practicas-atencion-implementar-aula>. (Consultados el 13/06/2020)

81. Bruer JT. Education and the brain: a bridge too far. Educ. Res. 1997; 26, 4-16. doi: 10.3102/0013189X026008004. 